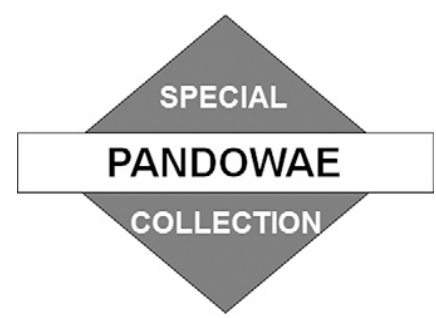

\title{
Upscale Error Growth in a High-Resolution Simulation of a Summertime Weather Event over Europe*
}

\author{
Tobias Selz AND GeORge C. CRAIG \\ Meteorologisches Institut, Ludwig-Maximilians-Universität, München, Germany
}

(Manuscript received 1 May 2014, in final form 19 September 2014)

\begin{abstract}
The growth of small-amplitude, spatially uncorrelated perturbations has been studied in a weather forecast of a 4-day period in the summer of 2007, using a large domain covering Europe and the eastern Atlantic and with explicitly resolved deep convection. The error growth follows the three-stage conceptual model of Zhang et al., with rapid initial growth ( $e$-folding time about $0.5 \mathrm{~h}$ ) on all scales, relaxing over about $20 \mathrm{~h}$ to a slow growth of the large-scale perturbations ( $e$-folding time $12 \mathrm{~h}$ ). The initial growth was confined to precipitating regions, with a faster growth rate where conditional instability was large. Growth in these regions saturated within 3-10 h, continuing for the longest where the precipitation rate was large. While the initial growth was mainly in the divergent part of the flow, the eventual slow growth on large scales was more in the rotational component.

Spectral decomposition of the disturbance energy showed that the rapid growth in precipitating regions projected onto all Fourier components; however, the amplitude at saturation was too small to initiate the subsequent large-scale growth. Visualization of the disturbance energy showed it to expand outward from the precipitating regions at a speed corresponding to a deep tropospheric gravity wave. These results suggest a physical picture of error growth with a rapidly growing disturbance to the vertical mass transport in precipitating regions that spreads to the radius of deformation while undergoing geostrophic adjustment, eventually creating a balanced perturbation that continues to grow through baroclinic instability.
\end{abstract}

\section{Introduction}

Predictability of the atmosphere is intrinsically limited as a result of the exponential growth of small-amplitude errors. The time interval for which skillful predictions are possible is, however, strongly dependent on spatial scale (Lorenz 1969). Baroclinic instability associated with the equator-to-pole temperature gradient drives synoptic-scale motions while conditional instability associated with an unstable vertical profile is a major energy source on the cloud scale. Error growth processes in the atmosphere can be related to these two primary instabilities and occur on corresponding scales. Hohenegger and Schär (2007a)

\footnotetext{
* Supplemental information related to this paper is available at the Journals Online website: http://dx.doi.org/10.1175/MWR-D14-00140.s1.

Corresponding author address: Tobias Selz, Meteorologisches Institut, Theresienstrasse 37, 80333 München, Germany. E-mail: tobias.selz@lmu.de
}

showed that error growth rates of synoptic-scale systems and convective systems differ by roughly a factor of 10 , as did the time to saturation of error amplitudes.

Exponential error growth in baroclinic systems can be related to the growth rate of the underlying system, but can be preceded by an adjustment phase where the growth rate is dependent on the structure and amplitude of the initial perturbation (Zhu and Thorpe 2006). In contrast, the errors on convective scales grow at the location of the convection (Zhang et al. 2007) and seem to be almost insensitive to the perturbation method (Hohenegger and Schär 2007b) (i.e., their amplitude and spatial structure). Even small-amplitude acoustic waves are sufficient to generate significant divergence of two simulations on the convective scale after about $10 \mathrm{~h}$. Larger sensitivity to perturbation amplitudes and structures may, however, originate from the boundary layer in an environment with high convective available potential energy (CAPE) and convective inhibition. Here perturbations might be able to trigger additional convective cells instead of just displacing existing ones (Leoncini 
et al. 2010). Furthermore there is evidence that the upscale impact of convection on the geostrophically balanced flow may also depend strongly on the convective regime (i.e., if the convective activity is mainly driven by large-scale forced ascent or strong local surface fluxes; Done et al. 2006).

Although the growth rate of small-scale errors is much higher than for synoptic-scale errors, they are also smaller in energy and saturate much faster than the slowergrowing errors on large scales. Further upscale growth is then much slower and the impact of the small-scale errors is reduced. On the other hand, the initial state uncertainty is much smaller at the synoptic scale, which might increase the importance of upscale-grown disturbances from convective uncertainty at longer forecast lead times (Rodwell et al. 2013). This is further supported by the fact that global ensembles forecast systems become underdispersive when only initial state uncertainty is sampled (Leutbecher and Palmer 2008), which also might be at least partly related to unresolved upscale processes from convection.

Zhang et al. (2007) recently investigated the intrinsic predictability of an idealized baroclinic wave using a convection resolving numerical model. They argued that the intrinsic predictability on synoptic scales is indirectly limited by upscale growth from the convectivescale errors and suggested a three-stage conceptual model of error growth. Stage 1 comprises fast small-scale error growth driven by convective instability and moist processes. These errors quickly saturate because of a complete displacement of individual convective cells. During stage 2, the transition stage, these convectivescale errors start to spin up balanced motions, which continue to grow at a slower rate through baroclinic instability (stage 3). This model describes how the intrinsic convective-scale uncertainty is able to grow upscale and contaminate the mesoscale and the large-scale forecast after a period of time.

However, the relevance of this conceptual model is arguable because of the high level of idealization. The $10-\mathrm{km}$ model resolution used in the main experiments of Zhang et al. (2007) was marginal for simulating convection, and the absence of surface fluxes may strongly influence error growth properties on the convective scale. This latter issue also applies to their short-time experiment with a nested domain at $3-\mathrm{km}$ resolution. With the increase in computational power in recent years, it is now practical to simulate weather systems at uniformly high resolution in a full-physics numerical model over length scales that include several times the Rossby radius of deformation. The aim of this paper is to evaluate whether the three stage conceptual model of Zhang et al. (2007) can be applied to error growth in a realistic weather event, and if possible, to quantitatively characterize the three stages. In addition to growth rate diagnostics, the study will analyze spectra of total and perturbation kinetic energy and introduce a Rossby number diagnostic to measure the development of geostrophic balance. The impact of the diurnal cycle will also be investigated, and the role of gravity waves radiating from convective regions in upscale growth or errors will be discussed.

\section{Experimental design}

This study employs a nonhydrostatic limited-area atmospheric model provided by the Consortium for SmallScale Modeling (COSMO) (Baldauf et al. 2011). It has been applied for many years by the German Meteorological Service [Deutscher Wetterdienst (DWD)] for operational forecasting with a $7-\mathrm{km}$ grid spacing (COSMO-EU) and a $2.8-\mathrm{km}$ grid spacing (COSMO-DE). In the higherresolution configuration deep convection is considered to be resolved and the model is run with only a parameterization for shallow convection. The present study is based on the COSMO-DE configuration, but with the domain enlarged to a size of about $7000 \mathrm{~km}$ by $4250 \mathrm{~km}$ $(2481 \times 1521$ grid points), which covers several times the Rossby radius of deformation. Some minor changes to the model setup were necessary since we used Integrated Forecast System (IFS) analysis and forecasts from the European Centre for Medium-Range Weather Forecasts (ECMWF) to provide the initial and boundary conditions whereas DWD uses their own global model. As at DWD the interpolation from the global model was not done directly, but a 7-km version of COSMO was used as intermediate step. Note that COSMO uses a rotated spherical grid, which in our setup is centered at $50^{\circ} \mathrm{N}, 10^{\circ} \mathrm{E}$.

We focus on the period 19-23 July 2007 in which an almost stationary low pressure system over Great Britain caused several days with intense convective activity and a cold front passage over central Europe (Fig. 1). This event was also part of the Convective and Orographically Induced Precipitation Study (COPS; Wulfmeyer et al. 2011) and will be discussed in more detail in the following section. COSMO simulations were started at 0000 UTC 19 July 2007 with initial and boundary conditions derived from the ECMWF analysis and deterministic forecast. We first run the high-resolution simulation for $96 \mathrm{~h}$ without any perturbation and refer to this experiment as the control run $(\mathrm{Ctl})$. In addition we carried out four perturbed runs, where a perturbation was applied to the model state at a single time $15,21,27$, and $33 \mathrm{~h}$ after forecast start, respectively. We refer to these experiments as P15, P21, P27, and P33. They thus sample one whole diurnal cycle. The first perturbation is applied $15 \mathrm{~h}$ after the start of the simulation to ensure that the error growth process is not contaminated by model spinup. 
0000 UTC 20 July 2007, flt $=24 \mathrm{~h}$

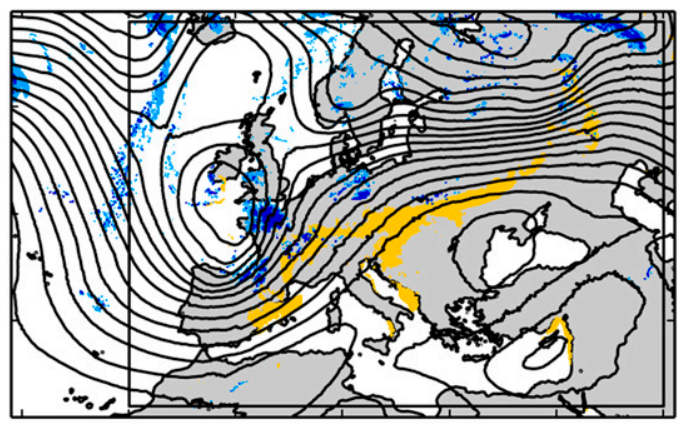

0000 UTC 22 July 2007, flt $=72 \mathrm{~h}$

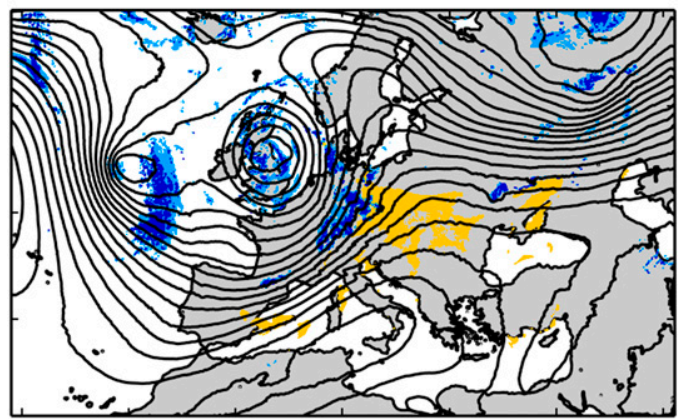

0000 UTC 21 July 2007, flt $=48 \mathrm{~h}$

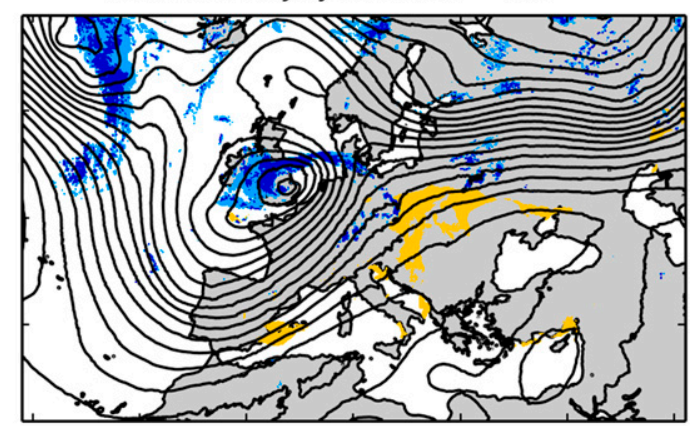

0000 UTC 23 July 2007, flt $=96 \mathrm{~h}$

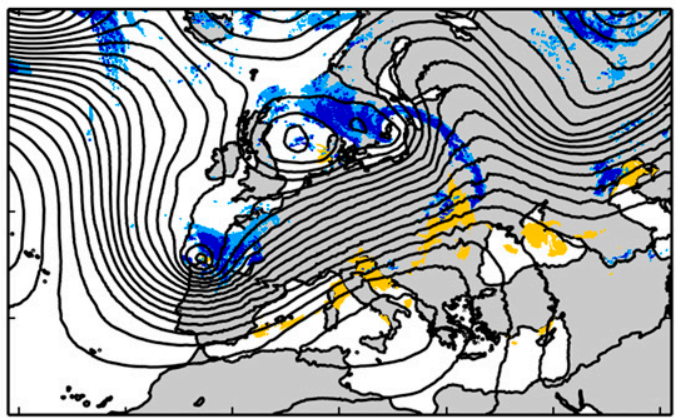

FIG. 1. General weather situation during the perturbation experiments. The plots show the unperturbed control run at 24-, 48-, 72-, and 96-h forecast lead time (flt). Initialization time was 0000 UTC 19 Jul 2007. Black lines show the 500-hPa geopotential with a line spacing of $250 \mathrm{~m}^{2} \mathrm{~s}^{-2}$. Light and dark blue shading show the precipitation rate above 0.1 and $1 \mathrm{~mm} \mathrm{~h}^{-1}$, respectively. Yellow shading indicates CAPE values above $500 \mathrm{~J} \mathrm{~kg}^{-1}$. (top left) The axis tick spacing is $10^{\circ}$ or approximately $1100 \mathrm{~km}$. The box indicates the horizontal extent of the domains $D_{1}$ and $D_{2}$ (which differ only in vertical extent).

As perturbation we used Gaussian-distributed, uncorrelated grid-scale noise with zero mean and a standard deviation of $0.01 \mathrm{~K}$, which was added to the temperature field on all model levels and all over the domain. For all four perturbed runs the same perturbation field was used. We decided to perturb the whole model domain to avoid complications from remote error growth triggering by sound waves as described in Hohenegger and Schär (2007b). After that perturbation, the model was run free without any further intervention. Boundary conditions are not perturbed and are identical in all five simulations.

The perturbations are not meant to represent an uncertainty in the initial conditions or an error from measurements, which would suggest much larger amplitudes and spatial correlations. By using white noise we also avoid introducing any particular spatial scale into the simulations. This design of the perturbations is very similar to Zhang et al. (2007). However, the noise amplitude is a factor of 20 smaller than in the earlier study to avoid strong initial decay of the perturbations near the grid scale due to numerical diffusion. The perturbations have been designed to set off the models phase space trajectory a tiny bit, generating five different realizations of the same meteorological conditions. The predictability that results from this kind of uncertainty, which is present even in a perfect model with (almost) perfect initial conditions is referred to as intrinsic predictability (Lorenz 1996; Melhauser and Zhang 2012).

Animations of the control run and the error growth experiments are available online as supplemental material. We will occasionally refer to these animations in the text.

\section{Weather situation}

An overview of the weather situation for the time period from 19 July 2007 to 23 July 2007 is presented first, based on the unperturbed control simulation. Qualitatively the COSMO forecast resembled the actual development, but a detailed comparison with observations has not been performed and is not the main focus of our current work. The general weather situation was characterized by an almost stationary low pressure system located over Great Britain (Fig. 1). Moist and unstable air was advected from the Atlantic to central Europe, further destabilized by synoptically forced ascent. Additionally the southern and eastern parts of Europe were already covered with a warm and potentially unstable air mass 


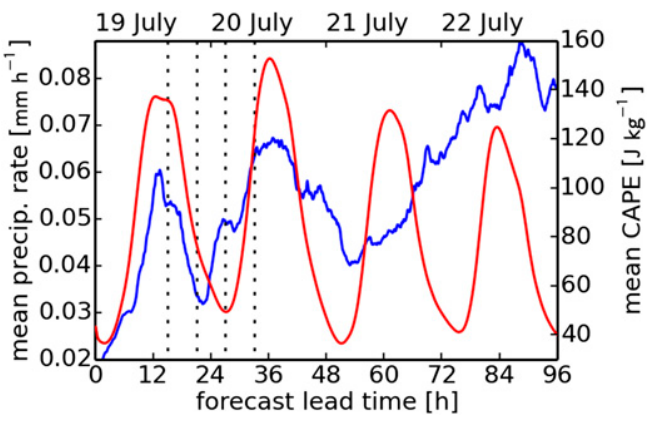

FIG. 2. Time evolution of precipitation rate (blue line, left axis) and CAPE (red line, right axis) of the unperturbed run, spatially averaged over domain $D_{1}$. The vertical dotted lines indicate the onsets of the four perturbation experiments.

resulting in very high values of CAPE. This led to several successive days of heavy precipitation over central Europe and the British Isles.

The domain-averaged precipitation rate and CAPE as function of forecast lead time is given in Fig. 2. After spin up the precipitation rate reached its first maximum in the afternoon of 19 July. During 20 July a cold front formed over Spain and western France, which developed into an intense and narrow squall line, crossing France and Germany and finally decaying over the North Sea at early on 21 July. In the course of that day convection formed again over Germany and was advected to the east. This convection persisted during the following night and developed again into a large front, reaching Russia and the Baltic states at the end of 22 July. In addition to Fig. 1 an animation of the unperturbed run is available in the online supplemental material, which gives an even better overview over the meteorological conditions and their evolution.

At $500 \mathrm{hPa}$ a distinct low was present over Great Britain during the whole forecast period (Fig. 1). After a slight increase of its geopotential during the first $29 \mathrm{~h}$ to $55200 \mathrm{~m}^{2} \mathrm{~s}^{-2}$ the geopotential fell roughly linearly in time and reached a minimum of $53700 \mathrm{~m}^{2} \mathrm{~s}^{-2}$ about $78 \mathrm{~h}$ after the forecast start. The upper-level trough over Great Britain was not associated with a distinct minimum in mean sea level pressure in the first $20 \mathrm{~h}$ of the simulation (not shown). Between 20 and $40 \mathrm{~h}$ a surface low developed and its central pressure decreased from about 1015 to $999 \mathrm{hPa}$. After that the central pressure remained more or less constant, varying between 998 and $1001 \mathrm{hPa}$.

\section{Diagnostics}

Before presenting the results we first introduce our main diagnostic tools, the difference total energy (partitioned by length scale), the kinetic energy spectrum of the difference flow, and finally the Rossby number of the difference flow. Because of the fixed lateral boundary conditions differences between the experiments are suppressed toward zero near the boundaries. In addition the strong inflow at the western boundary advects these zero differences farther into the domain and damps the error growth. For this reason we have chosen to analyze our data on a subdomain where we omitted $1^{\circ}$ or 40 grid points at the southern, eastern, and northern boundary and $11^{\circ}$ or 440 grid points at the western boundary. This subdomain then has a size of $5600 \mathrm{~km} \times 4000 \mathrm{~km}$ (see the box in Fig. 1).

In the vertical the model is configured with a damping layer at the top to suppress artificial reflections of vertically propagating gravity waves. This layer also damps any error growth in that region and we, therefore, additionally omitted the 14 uppermost model levels. The highest model level included has a mean height of $10.2 \mathrm{~km}$. We refer to this (three dimensional) subdomain as $D_{1}$. To be able to exclude the boundary layer and most orographic effects in some diagnostics we define a second domain, $D_{2}$, additionally omitting all model levels with a mean altitude smaller than $3 \mathrm{~km}$ (i.e., model levels $31-50$ ). Thus, note that the domains $D_{1}$ and $D_{2}$ differ only in vertical extent.

\section{a. Difference total energy}

The difference total energy (DTE) is defined following Zhang et al. (2003) as

$$
\operatorname{DTE}(\mathbf{x}, t)=\frac{1}{2}\left(\Delta u^{2}+\Delta v^{2}+\frac{c_{p}}{T_{r}} \Delta T^{2}\right),
$$

where the $\Delta$ indicates the difference of $u, v$, and $T$ between a perturbation experiment and the unperturbed control run; $c_{p}$ is the heat capacity of dry air at constant pressure; and $T_{r}=287 \mathrm{~K}$ is a reference temperature. To analyze error growth at different spatial scales a spectral filtering can be applied to $\Delta u, \Delta v$, and $\Delta T$ before calculating (1). Further following Zhang et al. (2007) we define three different scale ranges: a small scale $(S)$ up to $200 \mathrm{~km}$, a medium scale from 200 to $1000 \mathrm{~km}$, and a large scale from $1000 \mathrm{~km}$ up to the domain size. Scales are separated by truncating in wavenumber space. A domain integration of DTE provides a simple $L^{2}$ norm for the difference between a perturbed and the unperturbed run. It is defined by

$$
\operatorname{diDTE}(t)=\frac{1}{V\left[D_{1}\right]} \int_{D_{1}} d V \operatorname{DTE}(\mathbf{x}, t) .
$$

Here $V\left[D_{1}\right]$ indicates the volume of domain $D_{1}$, which vertically covers the whole troposphere. Figure 3 shows the perturbation lead time evaluation of diDTE for the 


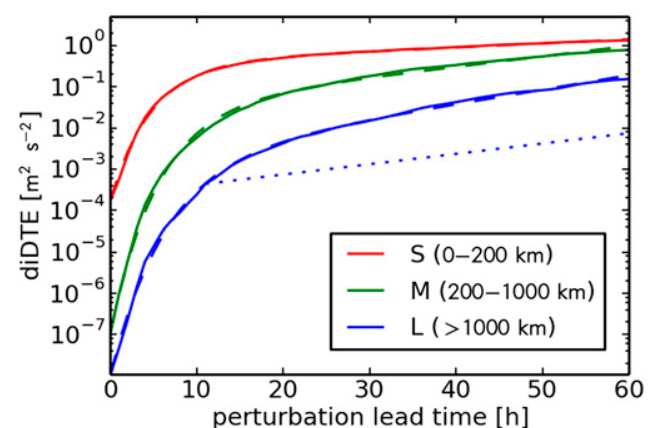

FIG. 3. Time evolution of diDTE for three different scales (solid lines), averaged over all four perturbation experiments. The dashed lines show a logarithmic least squares fit to (4). The dotted line indicates the extrapolation of the large-scale error at the end of stage 1 with a typical synoptic error growth rate. See text for details.

different scales, averaged over the four perturbation experiments.

To quantify the error growth we attempt to reproduce the shape of the lines using a minimally simple mathematical function: we first assume exponential growth at a fast growth rate $r$. This growth rate then decays exponentially toward zero at some rate $s$, corresponding to a complete saturation of the initial error growth. Finally an additional factor is added that describes a slow exponential growth at rate $g$ that will dominate for late perturbation lead times. We expect that $r \gg s \gg g$. The time-dependent growth rate, which is defined as the time derivative of the logarithm of (2), thus reads as

$$
\frac{d}{d t} \log (\mathrm{diDTE}) \approx r \exp (-s t)+g,
$$

which leads to the following function for domain-integrated DTE starting from an initial value $d_{0}$ at $t=0$ :

$$
\operatorname{diDTE}(t) \approx d_{0} \exp \left\{\frac{r}{s}[1-\exp (-s t)]\right\} \exp (g t)
$$

At early perturbation lead times $t \ll s^{-1}$ the initial growth will occur at the fast growth rate $r$ (since $r \gg g$ ), while the growth rate approaches $g$ for $t \gg s^{-1}$. We determine the parameters $r, s$, and $g$ from the DTE measurements by applying a least squares fit to the difference of the logarithms. The parameter $d_{0}$ is set to the initial value, which is directly determined by the initial noise. The dashed lines in Fig. 3 show the results of the fit for the three different spatial scales $S, M$, and $L$. The reciprocals of the rates correspond to $e$-folding times, which are given in Table 1 .

\section{b. Kinetic energy spectra}

A more detailed picture of scale interactions in error growth can be gained by looking at spectra since they
TABLE 1. Results of the logarithmic least squares parameter fit of the ensemble mean of diDTE to the function in (4) given in hours against the scale. The parameter $d_{0}$ was set to the initial value and is not displayed. The columns $S$ wet and $S$ dry show results for small scales when the domain integral of the DTE in (2) is split into a precipitating and a nonprecipitating area.

\begin{tabular}{llllll}
\hline & $S$ & $M$ & $L$ & $S$ wet & $S$ dry \\
\hline$r^{-1}$ & 0.61 & 0.44 & 0.53 & 0.34 & 0.70 \\
$s^{-1}$ & 4.7 & 5.5 & 6.4 & 3.5 & 5.1 \\
$g^{-1}$ & 44 & 17 & 12 & 290 & 39 \\
\hline
\end{tabular}

show each mode individually. Unlike Zhang et al. (2007) we do not consider spectra of DTE but kinetic energy spectra of the difference wind. The latter can be directly compared to the kinetic energy spectrum of the full model state.

For a given two-dimensional field $\psi(x, y)$ the Fourier transform is defined by

$\hat{\psi}\left(k_{x}, k_{y}\right)=\frac{1}{L_{x} L_{y}} \int_{0}^{L_{x}} \int_{0}^{L_{y}} d x d y \psi(x, y) \exp (i \mathbf{k} \cdot \mathbf{x})$,

with

$$
k_{x, y}=\Delta k_{x, y}\left(0, \pm 1, \pm 2, \ldots, \pm \frac{N_{x, y}}{2}\right) ; \quad \Delta k_{x, y}=\frac{2 \pi}{L_{x, y}},
$$

where $L_{x, y}$ indicates the length of the domain in the $x$ and $y$ directions, respectively. Since the Fourier transform requires periodic boundary conditions we apply the two-dimensional detrending described by Errico (1985) before calculating (5). This reduces the number of independent grid points in each direction by one, so that one row of points on the north and east boundaries of the domain are omitted in the application of (5). Despite the detrending there is still some aliasing from modes larger than the domain size to the largest resolved modes and caution is required when interpreting that part of the spectrum.

We calculate a one-dimensional power spectrum from (5) by integrating out the direction information. First we define the $k$ interval of the one-dimensional spectrum to be

$$
\Delta k=\max \left(\Delta k_{x}, \Delta k_{y}\right) .
$$

After that we sum the square of the absolute values of $\hat{\psi}$ over annuli of radius $k$ and thickness $\Delta k$ :

$$
\begin{aligned}
E_{\psi}(k) & =\frac{1}{\Delta k} \sum_{(k-\Delta k)^{2}<k_{x}^{2}+k_{y}^{2} \leq(k+\Delta k)^{2}} \hat{\psi} \hat{\psi}^{*} \quad \text { with } \\
k & =\Delta k \times 0,1, \ldots, N .
\end{aligned}
$$


The kinetic energy spectrum is then defined as

$$
E_{\mathbf{u}}(k)=\frac{1}{2}\left[E_{u}(k)+E_{v}(k)\right] .
$$

We can similarly apply (9) to the difference wind between a perturbed and the unperturbed run:

$$
E_{\Delta \mathbf{u}}(k)=\frac{1}{2}\left[E_{\Delta u}(k)+E_{\Delta v}(k)\right] .
$$

We evaluated the spectra on the subdomain $D_{2}$ to exclude boundary layer and orographic effects, meaning that we restricted the horizontal extent of the data before calculating (5) and then performing a vertical average of (9) and accordingly (10) over the free troposphere.

Spectra of kinetic energy of the COSMO-DE have been investigated by Bierdel et al. (2012) who applied the same method as described above. They have been shown to approximately reproduce a $-5 / 3$ slope. Since our domain is much larger we would also expect to resolve the mesoscale transition to a -3 slope. Indeed, the time-averaged kinetic energy spectrum of the control run (red line in Fig. 4) shows a distinct steepening of the slope at scales larger then about $100 \mathrm{~km}$.

In fact, the spectral energy density of the full model state in Fig. 4 is multiplied by a factor of 2 (which does not change the slope), so that it represents the saturation level for the kinetic energy spectra of the difference wind, which is plotted in Fig. 4 at several perturbation lead times, averaged over all four perturbation experiments. To see that this represents the saturation level, recall that the energy spectra of both the control and perturbed simulations follow the same (universal) spectrum. The spectrum of the difference wind will therefore be associated with phase shifts of the individual wavenumber components. A phase shift of $\pi$ leads to a maximum contribution in the difference and amplifies the mode by a factor of 4 (since the Fourier coefficients are squared). A random phase shift between 0 and $2 \pi$ will thus lead on average to an amplification by a factor of 2 .

\section{c. Rossby number}

To measure to what extend the perturbations are geostrophically balanced we define a Rossby number by considering horizontal divergence $(\Delta D)$ and vorticity $(\Delta \zeta)$ of the difference wind between a perturbed run and the control run. As for DTE we partition these fields into the three different scales and calculate a domain average of their squares:

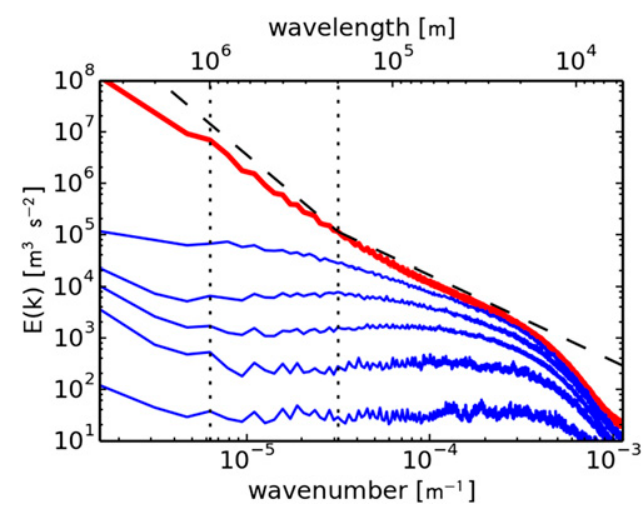

FIG. 4. Kinetic energy spectra of the control run (red line) and the difference between perturbed runs and control run for several perturbation lead times (blue lines). The control run spectrum is averaged from 12- to $96-\mathrm{h}$ forecast lead time and is multiplied by a factor of 2 to indicate the saturation level. The difference spectra are averaged over all four perturbation experiments. (from bottom to top) Difference spectra at 3, 6, 12, 24, and $60 \mathrm{~h}$ of perturbation lead time. The dashed black line indicates a $k^{-3}$ and $k^{-5 / 3}$ slope. The vertical dotted lines separate the three predefined scales $S, M$, and $L$.

$$
\|\Delta D, \Delta \zeta\|=\frac{1}{V\left[D_{2}\right]} \int_{D_{2}} d V \Delta D^{2}, \Delta \zeta^{2}
$$

Note that we again integrate over domain $D_{2}$ since we want to exclude the boundary layer in this balance diagnostic. A Rossby number can then be defined as the square root of the ratio of the divergence norm over the vorticity norm:

$$
\operatorname{Ro}=\sqrt{\frac{\|\Delta D\|}{\|\Delta \zeta\|}} .
$$

The result is shown in Fig. 5, again averaged over all four perturbation experiments.

\section{Results}

\section{a. Comparison to earlier studies}

Previous studies on convective-scale error growth (Zhang et al. 2003, 2007; Hohenegger and Schär 2007a; Leoncini et al. 2010) consistently showed a strong impact of moist processes on the growth rate of errors. The amplitude of growing perturbations differed significantly between areas with and without precipitation, and perturbations immediately decayed if latent heat release was set to zero.

To illustrate the early stage of error growth in our study we use the P33 experiment, which showed the fastest initial error growth of the four perturbation runs. In this experiment the first error growth phase coincides with the formation and eastward propagation of a cold front. 


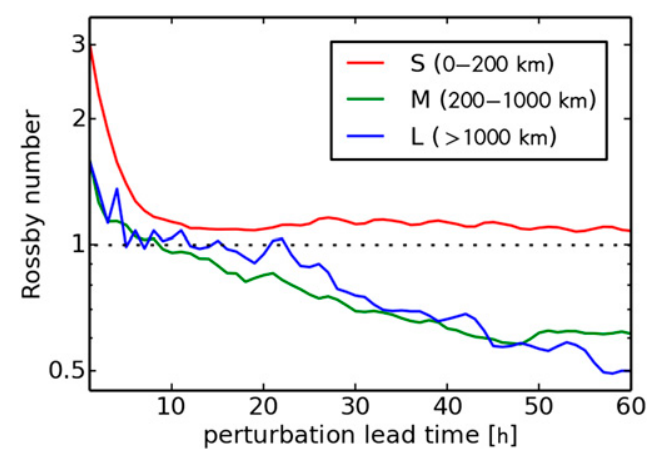

FIG. 5. The Rossby number in (12) over the perturbation lead time for the three different scales. Shown is the average over all four perturbation experiments.

Figure 6 shows the vertical wind speed difference at $500 \mathrm{hPa}$ of P33 minus the control run for four different perturbation lead times together with geopotential and precipitation rate from the control run. An animated version of the figure can be found in the supplemental material. As expected from the earlier studies, there is a clear correlation between the amplitude of the differences and the precipitation rate. Significant differences are almost entirely confined to precipitation areas, whereas in nonprecipitation areas their amplitude is several orders of magnitude smaller. Notably a decay of precipitation in a certain area also leads to a decay of errors, which can be seen in Fig. 6 by, for example, comparing errors and precipitation rates in the front at 12 - and 24 -h perturbation lead time. Despite such local effects, when integrated over the whole domain the errors grow monotonically (see below). Similar behavior is seen in each of the perturbation experiments.

From these small-scale perturbations, balanced difference patterns with much larger scale emerge. Figure 7 shows the low-pass-filtered 500-hPa geopotential difference for the P15 experiments at four different perturbation lead times. The online supplement includes animations of these fields for all four experiments, and also the logarithm of DTE at $500 \mathrm{hPa}$ (as shown in Fig. 11).

The time evolution of ensemble-averaged, domainintegrated DTE is shown in Fig. 3. The dashed lines in the figure show the logarithmic least squares fit of the DTE curves to the proposed function in (4). The deviation from the observed DTE curves is small and the fit seems to work very well over the full range of times. The parameters derived from the fit are given in Table 1 . The results show that the growth rate is highest in the first few hours then reduces to a much lower value that differs depending on the spatial scale. For the small scales the initial high growth rate reduces more rapidly and to a lower value than for the large scales, indicating saturation of the small-scale errors and persistent large-scale error growth at the end of the simulation. The initial gap between small- and large-scale errors of about four orders of magnitude is reduced to less than a factor of 10 over the $60 \mathrm{~h}$ of perturbation lead time (Fig. 3).

The perturbation growth is qualitatively very similar to that found by Zhang et al. (2007) (see their Fig. 7) and seems to fit the three stage conceptual model, although a closer inspection reveals some discrepancies. First the initial growth rate $r$ is about equal at all spatial scales, although the errors on large scales are not thought to be driven by convective instability. Second the final growth rate $g$ of the small-scale error is indeed lower than that of the large scale, however, a significant growth rate still remains. The imperfect saturation at scales below $200 \mathrm{~km}$ is even more evident in the kinetic energy spectrum diagnostic (Fig. 4). In addition the final large-scale error assessed in terms of the geopotential difference is about half than that found in Zhang et al. (2007) despite their shorter perturbation lead time. These differences are discussed further in section 6 .

\section{b. Initial error growth}

By fitting the proposed error growth function (4) to the time series of DTE we derive an estimate for the initial growth rate of the small-scale error. We find an $e$-folding time of $0.61 \mathrm{~h}$ (Table 1 ). Note that since DTE is a squared difference an $e$-folding time of a linear difference is twice as long (about 1.2 h). However, as seen in Fig. 6, the error growth rate in precipitating and nonprecipitating areas must be very different, meaning that the estimated growth rate of the domain-integrated errors is in fact an average of a slower dry error growth and a faster moist error growth.

To see this explicitly we divide the integration domain into precipitating and nonprecipitating subdomains using a threshold precipitation rate of $0.1 \mathrm{~mm} \mathrm{~h}^{-1}$ and integrate the small-scale filtered DTE separately. We refer to those quantities as conditionally integrated DTE (ciDTE). The result is given in Fig. 8 (black lines). Again the shape of the curves is very well fitted by (4) and the parameters $r^{-1}, s^{-1}$, and $g^{-1}$ can be estimated (two rightmost columns in Table 1). As expected we find different growth rates for dry $\left(r^{-1}=0.70 \mathrm{~h}\right)$ and wet $\left(r^{-1}=0.34 \mathrm{~h}\right)$ regions. The short $e$-folding time in the wet regions is consistent with the typical half-hour convective turnover time, while the initial growth rate in dry regions is about a factor of 2 slower.

To explore how moist processes influence error growth we further separate the DTE integration into four groups by distinguishing between heavy and weak precipitation (threshold $1 \mathrm{~mm} \mathrm{~h}^{-1}$ ) and low and high CAPE (threshold $10 \mathrm{~J} \mathrm{~kg}^{-1}$ ). The CAPE threshold is 
15 UTC 20 July 2007, plt $=6 \mathrm{~h}$

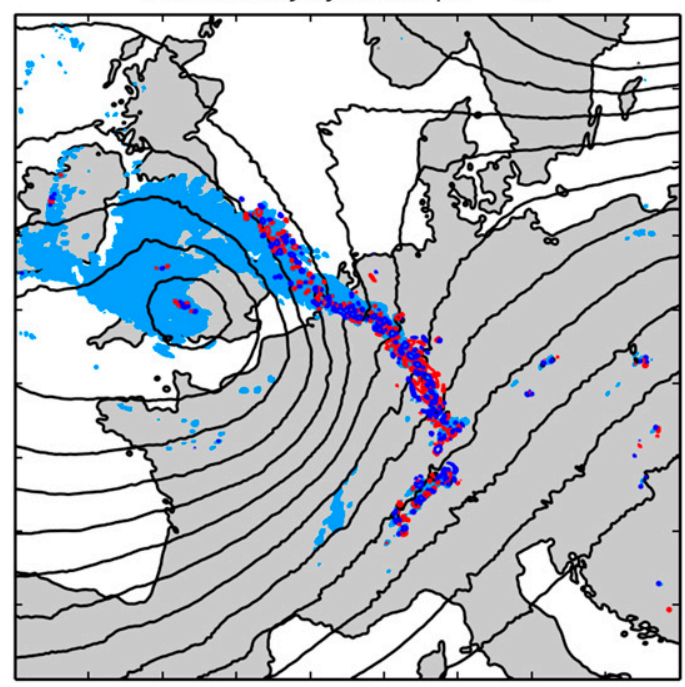

09 UTC 21 July 2007, plt $=24 \mathrm{~h}$

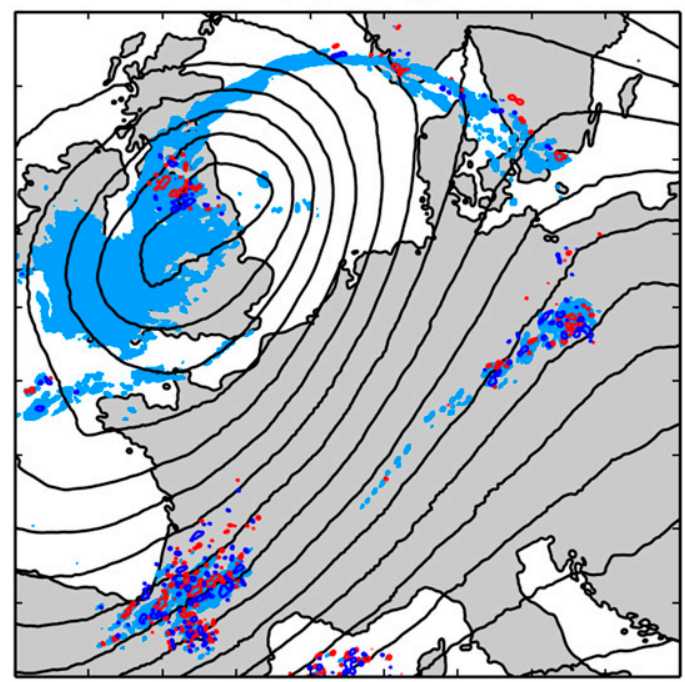

21 UTC 20 July 2007, plt $=12 \mathrm{~h}$

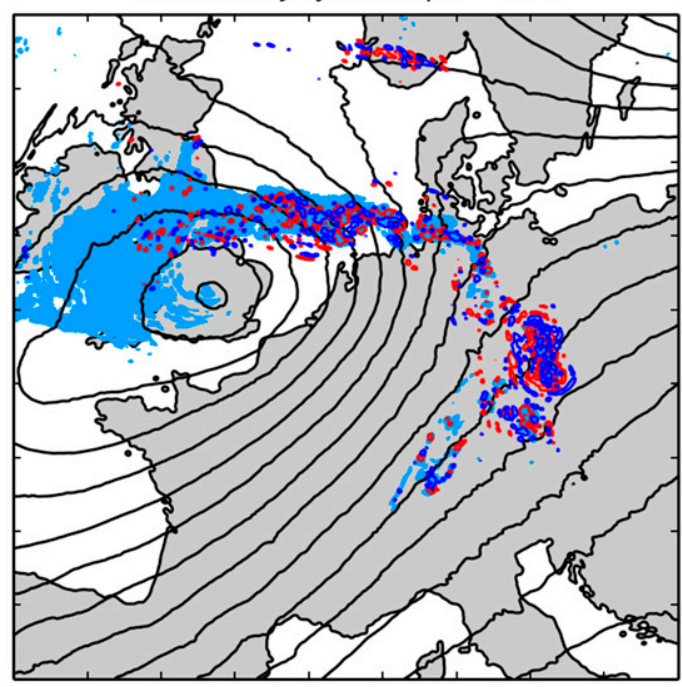

09 UTC 22 July 2007, plt $=48 \mathrm{~h}$

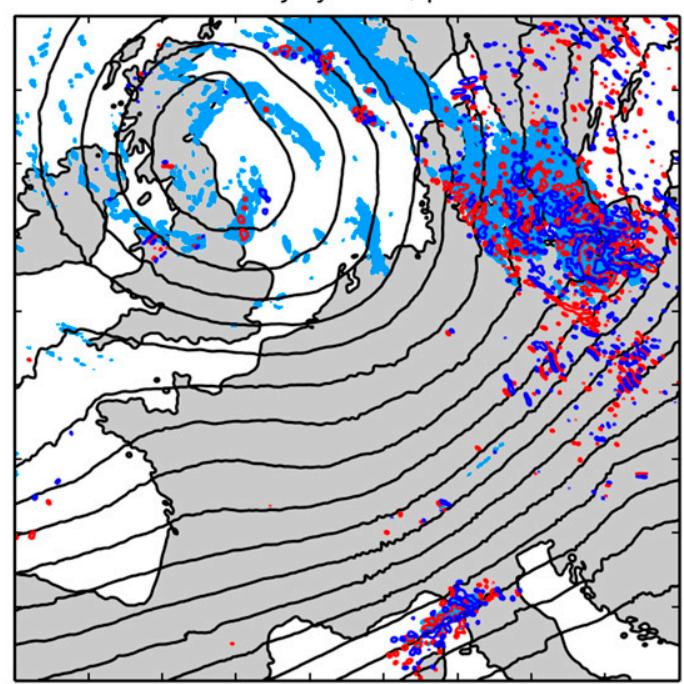

FIG. 6. Vertical wind difference (colored lines) between $\mathrm{P} 33$ and $\mathrm{Ctl}$ at different perturbation lead times (plt). The red and blue lines indicate a difference of 2 and $-2 \mathrm{~Pa} \mathrm{~s}^{-1}$, respectively. The black lines show the 500-hPa geopotential of the control run (line spacing $250 \mathrm{~m}^{2} \mathrm{~s}^{-2}$ ). Light blue shaded areas have a precipitation rate exceeding $0.1 \mathrm{~mm} \mathrm{~h}^{-1}$. The axis tick spacing is $2^{\circ}$ or approximately $220 \mathrm{~km}$.

chosen to separate between convective and stratiform precipitation. Since convection removes the CAPE locally it is the larger-scale environment of a convective cell that matters. Therefore, we removed scales below $200 \mathrm{~km}$ from the CAPE field before evaluating the threshold. The colored lines in Fig. 8 show the time dependence of DTE for these four groups. Table 2 lists their initial growth rate and saturation amplitude. The initial growth rate appears to be determined mainly by the instability: errors grow faster in more unstable (high CAPE) environments. The saturation amplitude on the other hand is related to the precipitation rate and thus to the rate of latent heat release. The differences among the different precipitating conditions are, however, much smaller than the difference between precipitating and dry areas showing that latent heat release is the main source for rapid small-scale error growth and further properties of this latent heat release are of second-order importance.

The modulation of the convective instability by surface fluxes leads to a very distinct diurnal cycle in the domain-averaged CAPE (Fig. 2). In addition the precipitation rate is also coupled to the diurnal cycle during the first two days. Thus, P15 and P33 are perturbed under much more unstable conditions than P21 and P27 
00 UTC 20 July 2007, plt $=24 \mathrm{~h}$

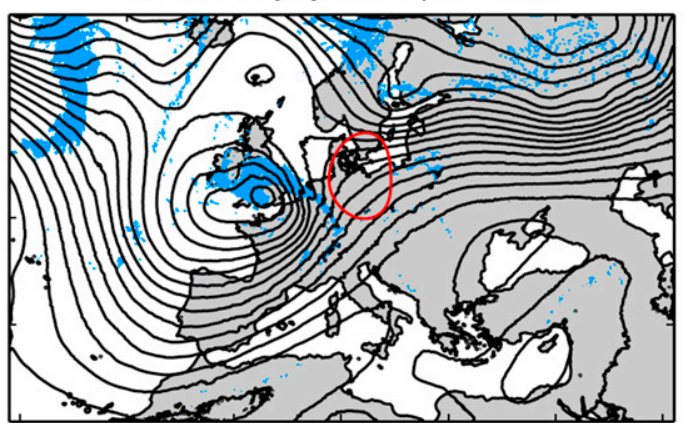

00 UTC 21 July 2007, plt $=48 \mathrm{~h}$

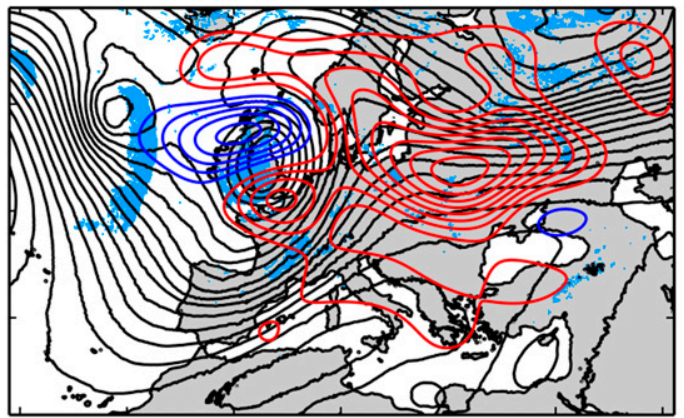

12 UTC 20 July 2007, plt $=36 \mathrm{~h}$

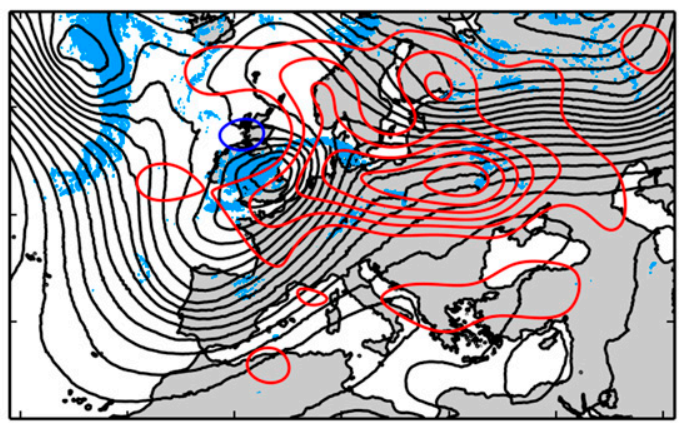

12 UTC 21 July 2007, plt $=60 \mathrm{~h}$

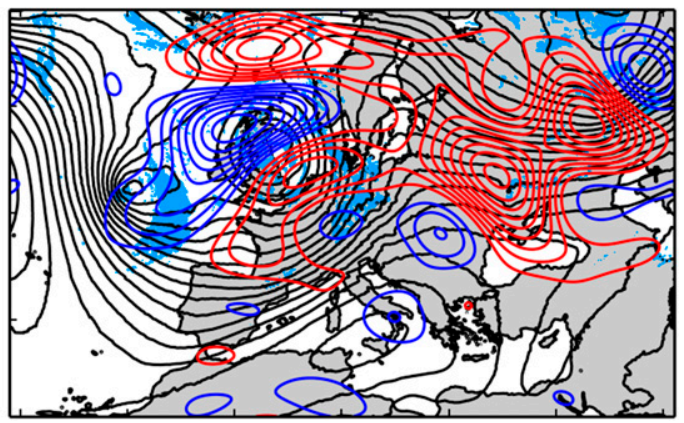

FIG. 7. Development of the large-scale geopotential difference of experiment P15, given at four different perturbation lead times (plt). The colored lines show the difference of 500-hPa geopotential between perturbed and unperturbed run with scales below $1000 \mathrm{~km}$ filtered out. Red lines indicate positive and blue lines indicate negative differences with a line spacing of $5 \mathrm{~m}^{2} \mathrm{~s}^{-2}$. The black lines show the 500-hPa geopotential of the control run with a line spacing of $250 \mathrm{~m}^{2} \mathrm{~s}^{-2}$. Light blue shading indicates areas where the control run precipitation rate exceeds $0.1 \mathrm{~mm} \mathrm{~h}^{-1}$. The axis tick spacing is $10^{\circ}$ or approximately $1100 \mathrm{~km}$.

and should, according to the results above, show faster error growth initially. It was also found in Zhang et al. (2006) that errors grow faster during the second half of the day due to an equivalent diurnal cycle of the convective instability. To confirm this we plotted the ratio of the small-scale diDTE from the four different perturbation experiments to their ensemble mean (Fig. 9), which indeed shows that the initial growth rate of P15 and P33 is much faster than the mean while it is clearly slower in P21 and P27. Furthermore for P15 the instability and precipitation rate strongly decreases right after the perturbations are introduced while they increase further for P33. Consistent with this the fast growth rate in P33 persists much longer than for P15. However, the initial differences in error growth are completely compensated after $15 \mathrm{~h}$ and all four experiments show about the same departure from the control run.

\section{c. Saturation of small-scale errors}

The fit of small-scale DTE to our mathematical model of the error development in (4) gives an estimate of $4.7 \mathrm{~h}$ for the parameter $s$, which is related to the time it takes the errors to saturate (more precisely for growth to slow down to the final rate $g$ ). The initial fast error growth rate is thus reduced by a factor $e^{-1}$ in about $5 \mathrm{~h}$. If we consider the small scales saturated when their fast growth rate $r$ has decreased to $5 \%$ of its initial value, this leads to an estimation of about $14 \mathrm{~h}$ for the corresponding time scale. However, as already stated above, the saturation is imperfect since the final growth rate $g$ is still positive.

This is different when looking at the wet region integral of DTE in Fig. 8 (black solid line). Here, saturation is very clear and the estimated final growth rate $g$ is essentially zero (Table 1). The fast growth rate $r$ is reduced by $95 \%$ in only $10.5 \mathrm{~h}$. In contrast the errors in dry regions (black dashed line in Fig. 8) do not show such a clear saturation but a small and fairly linear (rather than exponential) growth between 24 and $60 \mathrm{~h}$. Since, however, most grid points in the domain are dry, the dry error growth properties and the domain-integrated error growth properties are similar (Table 1). The domainintegrated DTE diagram only partly captures the properties of moist small-scale error growth.

The kinetic energy spectrum of the difference wind also reflects the domain-averaged error properties. Figure 4 shows that there is still significant growth even at very small scales between the 12 - and 60 -h perturbation lead time. At the small scale end of the spectrum 

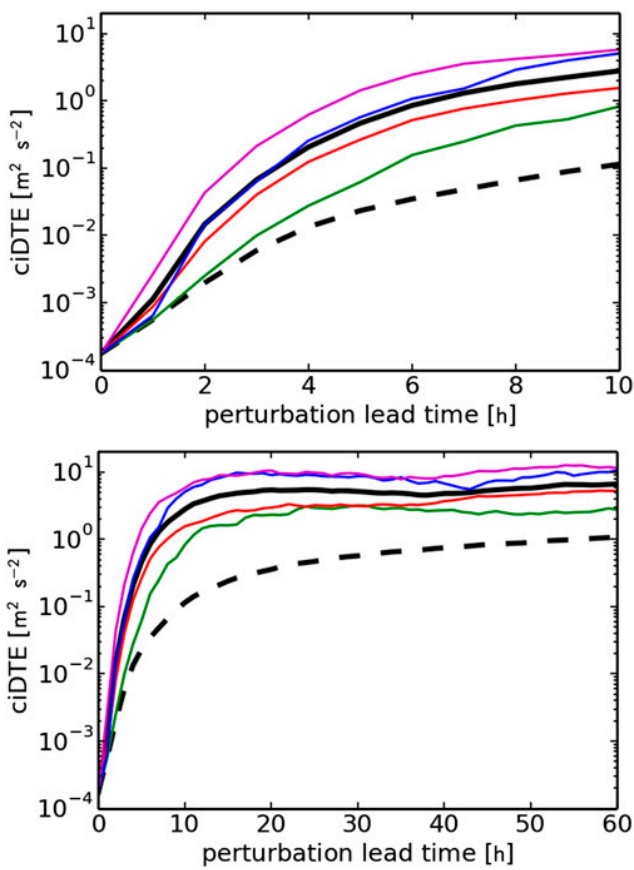

FIG. 8. Time evolution of conditionally integrated DTE (ciDTE), averaged over the four different perturbation experiments on small scales $(S)$. (top) The first $10 \mathrm{~h}$ and (bottom) the whole 60-h range. The different conditions are as follows: no precipitation (black dashed), precipitation (black), weak precipitation and low CAPE (green), weak precipitation and high CAPE (red), strong precipitation and low CAPE (blue), and strong precipitation and high CAPE (purple). Thresholds are given in the caption of Table 2.

and for long perturbation lead times the difference spectra almost exactly reach the saturation level (the actual amplification is between 1.7 and 1.8). However, at 60 -h perturbation lead time this is still only true for modes up to about $30-\mathrm{km}$ wavelength, which indicates that the error in dry regions still has room to grow further.

The clear saturation of errors in precipitating areas indicate that locally enhanced amplitudes of wind and temperature fluctuations in the presence of latent heat release and their complete or rather random displacement between the perturbed and the unperturbed run determine the saturation and thus the end of stage 1 . In our simulations this took about $11 \mathrm{~h}$ from the moment of perturbation. This time interval may, however, be sensitive to the noise amplitude and also to numerical diffusion and, thus, resolution. In contrast to precipitation areas, the errors in dry regions grow slowly but continuously and would most likely continue to grow beyond the 60 -h perturbation lead time. This presumably indicates a different physical mechanism from the rapid initial error growth, and the saturation in precipitating regions marks the end of the first stage of error growth in the conceptual model.
TABLE 2. Initial ciDTE $e$-folding time (top value, given in hours) and saturation amplitude (bottom value, given in $\mathrm{m}^{2} \mathrm{~s}^{-2}$ ) under four different conditions. Thresholds of 0.1 and $1 \mathrm{~mm} \mathrm{~h}^{-1}$ define nonprecipitating, weak, and strong precipitating grid points, respectively. A threshold of $10 \mathrm{~J} \mathrm{~kg}^{-1}$ is chosen to distinguish between low and high CAPE. The saturation amplitude was calculated by taking the time mean of ciDTE between 20 - and 60 -h perturbation lead time.

\begin{tabular}{lcc}
\hline \hline & Weak precipitation & Strong precipitation \\
\hline Low CAPE & 0.86 & 0.77 \\
& 2.7 & 8.3 \\
High CAPE & 0.63 & 0.37 \\
& 3.9 & 10.0 \\
\hline
\end{tabular}

\section{d. Properties of the large-scale growth}

An example of the development of the large-scale difference in the 500-hPa geopotential can be seen in Fig. 7. The patterns produced in the other three experiments show different structures, and there is considerable variability in amplitude of the large-scale perturbations, even though the start time of the perturbations in the different experiments is only $6 \mathrm{~h}$ apart in a slowly changing environment. We find root-mean-square differences of the $500-\mathrm{hPa}$ geopotential $60 \mathrm{~h}$ after perturbation of $11.2(\mathrm{P} 21)$, 13.3 (P33), 18.5 (P15), and $23.3 \mathrm{~m}^{2} \mathrm{~s}^{-2}$ (P27). To provide a reference for the magnitude of these differences we calculated the spatial and temporal mean of the $500-\mathrm{hPa}$ geopotential standard derivation of the ECMWF ensemble over the considered domain and time period at 6-h forecast lead time and obtained a value of about $45 \mathrm{~m}^{2} \mathrm{~s}^{-2}$. Thus, the amplitude of the initially tiny perturbations after $60 \mathrm{~h}$ in our experiments reaches almost half the spread in a 6-h ensemble forecast started with estimated observational uncertainty.

The final large-scale amplitude of the perturbations in the different experiments does not correlate with the initial growth rate or saturation amplitude of the small-scale perturbations. The observed variability in the final largescale difference is probably due to a basically random projection of the growing perturbation onto the leading Lyapunov vectors of the large-scale dynamics. These projections may depend on subtle variations in the position of the perturbation relative to the phase of the largescale wave. We expect that runs with different realizations of the noise but fixed perturbation time would lead to a similar variability. However, verification is beyond our current computing capabilities.

As previously noted the initial large-scale growth rate is similar to the small-scale rate (Fig. 3 and Table 1) and thus much higher than the expected growth rates for synoptic-scale dynamics. Since the large-scale growth rate is defined by a cutoff scale in Fourier space, the explanation for this rapid growth can be found by considering 


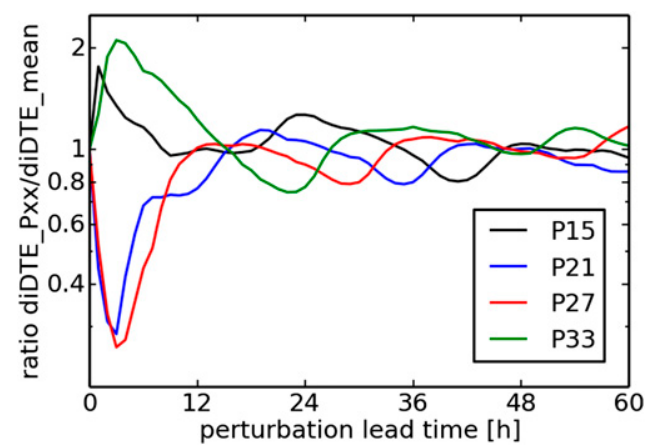

FIG. 9. Ratios of diDTE from the four different perturbation experiments to their mean on small scales $(S)$.

the kinetic energy spectrum of the difference wind. The initial error growth is confined to precipitating regions, and is, therefore, highly intermittent in space. The spectral decomposition of a Gaussian function was investigated analytically in Durran et al. (2013) [see their Eq. (1)]. The result is another Gaussian function centered at $k=0$, which means the spectral decomposition of an isolated feature projects onto all scales and most prominently onto the largest scales. In addition, the widths of the spatial and the spectral Gaussian are inversely proportional to one another. Note that in the double-logarithmic representation such a Gaussian appears flat at large scales and then drops of pretty sharp at the scale of its spatial counterpart. Qualitatively, such a pattern is clearly seen in Fig. 4 for perturbation lead times up to $24 \mathrm{~h}$, meaning that the initial growth for the medium and large scales can be interpreted as a projection of small-scale perturbation growth onto larger scales. Note that the very largest scales in Fig. 4 are influenced by the nonperiodicity of the domain, and the increased energy levels may be artifacts of the calculation.

Looking at the medium scales (the scales between the two dotted vertical lines in Fig. 4) the slope of the difference spectra seems to change significantly for perturbation lead times exceeding $24 \mathrm{~h}$ from a horizontal or slightly increasing slope to a clearly decreasing slope at $60 \mathrm{~h}$. To see this explicitly we calculated the linear regression coefficients of the difference spectra in the medium wavenumber range at hourly intervals. The time evolution of the slope is shown in Fig. 10. There are some very large fluctuations at early perturbation lead times (especially 4 and $5 \mathrm{~h}$ ), which are most likely an artifact of the detrending procedure. Nevertheless, the steady decrease of the slope after about the 24-h perturbation lead time is very clear. This change in the spectral slope in the medium range indicates that now a significant large-scale component in the difference field emerges, which can no longer be explained by a projection of small-scale disturbances.

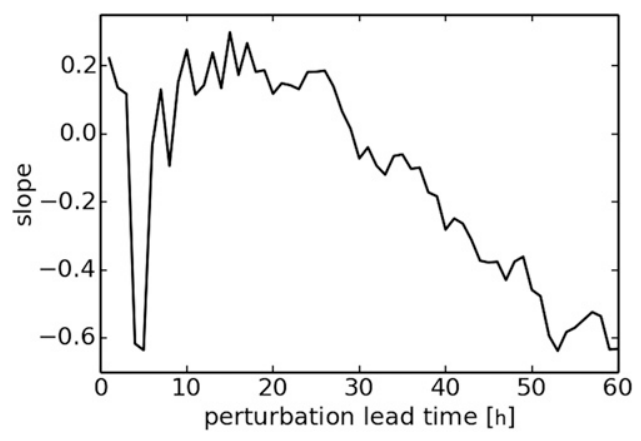

FIG. 10. Slope of the double-logarithmic kinetic energy spectrum of the difference wind over perturbation lead time. The slope is calculated from the averaged spectrum over all four perturbation experiments between $200-$ and $1000-\mathrm{km}$ wavelength (scale $M$, indicated by the two dotted vertical lines in Fig. 4).

To further confirm this picture we finally consider the Rossby number diagnostic [(12) and Fig. 5]. Since the balanced, quasigeostrophic dynamics on large scales are dominated by rotation and are nearly nondivergent, their Rossby number in (12) should be well below 1 . Using the wind field of the control run in (12) we find a mean value of 0.12 , as expected. The Rossby number of the large-scale difference wind (blue line in Fig. 5), however, is greater than or equal to 1 at early perturbation lead times, meaning that the differences are not yet geostrophically balanced. Furthermore, the time development of the large- and medium-scale Rossby number is initially similar to the small-scale Rossby number, which also indicates that we just see a projection of small-scale disturbances driven by convective instability and latent heat release onto the large scales. Consistently with the steepening of the spectral slope, after about $24 \mathrm{~h}$ the large-scale Rossby number starts to drop significantly below 1 , in contrast to the Rossby number of the small-scale flow. This indicates the emergence of a growing balanced perturbation that comes to dominate over the unbalanced projection from small scales and coincides with the change in spectral slope at the medium scales. This transition marks the beginning of the third stage of the conceptual model.

\section{e. The role of mesoscale processes}

While the end of stage 1 and the onset of stage 3 of the conceptual error growth model could clearly be identified by the diagnostics shown above, the identification of the transition phase (stage 2) and its physical mechanism are less clear. This stage will probably not just lie in between stage 1 and 3 because the transition may well begin before the saturation is complete. In addition, there is probably a constant and ongoing transition and adjustment process of small-scale differences after their saturation, which 

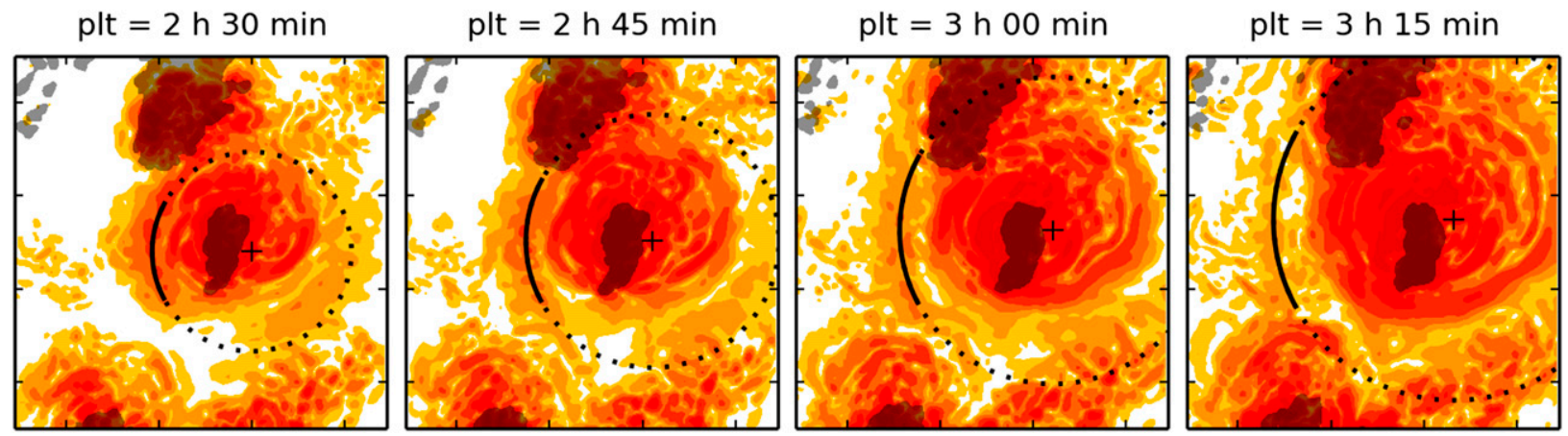

FIG. 11. Logarithm of 500-hPa DTE of the P15 experiment (reddish colors) on a subdomain around a convective cell for four different perturbation lead times (plt). The dark shading indicates areas of precipitation $\left(>0.1 \mathrm{~mm} \mathrm{~h}^{-1}\right)$. The black dotted circle is chosen to match approximately the circular pattern on the west side of the convective cell in the leftmost figure (solid line). Its center (the black cross) is advected with the horizontal wind (small scales filtered out) and its radius is increased by an estimate of the gravity wave speed of $36.0 \mathrm{~m} \mathrm{~s}^{-1}$. The axis tick spacing is $1^{\circ}$ or approximately $110 \mathrm{~km}$.

eventually becomes negligible compared to the growing synoptic-scale difference.

In principle a mechanism for growth of perturbations on the mesoscale is not required for the development of a large-scale perturbation. The saturated small-scale errors will directly project onto the large-scale motions and excite baroclinic error growth. However, if we extrapolate the projected energy at saturation time (about $11 \mathrm{~h}$ ) with a typical baroclinic growth rate (a geopotential doubling time of a day, which corresponds to a DTE $e$-folding time of $17.3 \mathrm{~h}$ ) the final large-scale DTE amplitude is more than an order of magnitude below the observed value (dotted line in Fig. 3). That shows that an efficient transition through the mesoscale is able to speed up the largescale error growth and significantly reduce the intrinsic predictability of the synoptic-scale flow.

The question remains what physical processes are most important for the transition processes in the mesoscale and the emergence of geostrophic balance. Initial rapid perturbation growth is confined to precipitating regions, which are also regions of ascent and upper-level divergence and are associated with spreading gravity waves (Bretherton and Smolarkiewicz 1989). The initial perturbations shift the convective cells and their associated circulations (Fig. 6). As shown in Fig. 11, and especially in the animations provided in the online supplement, the perturbed region expands outward from the precipitation location in a form that visually resembles a gravity wave front. It would be expected that as this divergent perturbation expands to the Rossby radius of deformation, a rotational component would spin up, leading to a geostrophically balanced perturbation. While it is difficult to prove this scenario rigorously, we will attempt to show that the speed and duration of the spatial growth is consistent with the hypothesis.
First, we consider the spread of perturbations from a selected convective cell from the P15 experiment. The propagation speed of a gravity wave is estimated by assuming the hydrostatic nonrotating regime, which leads to $c=N / m$ (Gill 1982), where $N$ is the Brunt-Väisälä frequency and $m$ is the vertical wavenumber. Note that in this limit the gravity waves are nondispersive. We further assume a deep, troposphere-filling gravity wave and thus set the vertical wavenumber to $2 \pi$ over twice the tropopause height $H$, which results in a propagation speed of

$$
c=\frac{N H}{\pi} \text {. }
$$

In the leftmost plot of Fig. 11 a wave front is selected and is matched with a circle. The gravity wave speed in (13) is estimated by calculating a mean Brunt-Väisälä frequency over the subdomain shown in the figure and with $H=$ $10 \mathrm{~km}$ leads to $c=36.0 \mathrm{~m} \mathrm{~s}^{-1}$. The radius of the circle is then increased with that speed and its center is advected with the large-scale wind field. It can be seen from the figure that the circle matches the propagating wave front closely for about an hour. After that, the wave becomes difficult to distinguish from surrounding anomalies.

Second we consider a dimensional argument for the time required for gravity waves to generate balanced perturbations. The time scale for this process to happen can be estimated roughly by the time the gravity waves need to travel the Rossby radius of deformation $r_{D}=$ $N H / f$. Together with (13) we find a simple and universal expression for this transition time scale:

$$
t_{T}=\frac{r_{D}}{c}=\frac{\pi}{f}
$$

which equals $7.8 \mathrm{~h}$ using the Coriolis parameter at $50^{\circ} \mathrm{N}$. A time scale of $\mathcal{O}(1 / f)$ for the error transition was also 
hypothesized by Zhang et al. (2007). It takes about $2 \mathrm{~h}$ for the initial perturbations to grow to the point where the first waves appear in the difference field (see Fig. 11 or supplemental animations), giving a time interval of roughly $10 \mathrm{~h}$ from the perturbation until the first indications of geostrophic balance should have developed. Figure 5 confirms that it takes indeed about $10 \mathrm{~h}$ until the development of the medium-range Rossby number starts to differ from the small-scale Rossby number and drops below one. The large-scale Rossby number drops even later, about $24 \mathrm{~h}$ after perturbations were introduced. At this stage, large-scale baroclinic growth can contribute strongly to the increase in disturbance energy. The estimates presented in this section are suggestive, but are still not a proof of the dominant role of gravity waves in the transition phase. A definitive result will probably require the development of new diagnostics that capture the space-time relationships of error growth.

\section{Summary and discussion}

We have simulated upscale error growth with the COSMO model in a real summertime weather event on a domain that covers several times the Rossby radius of deformation and has, at the same time, a resolution high enough to make a convection scheme dispensable. Since we used small-amplitude $(\sigma=0.01 \mathrm{~K})$ grid-scale noise perturbations on the temperature field our error growth experiments address the intrinsic predictability of the atmosphere (i.e., to what extent and by what processes forecasts are limited under the assumption of a perfect model and almost perfect initial conditions).

The results from our experiments are qualitatively similar to previous studies (Zhang et al. 2003; Hohenegger and Schär 2007a) and confirm the three error stage conceptual model suggested by Zhang et al. (2007). This consists of a first stage, where errors grow rapidly on small scales in the presence of convective instability and latent heat release, but saturate quickly. This is followed by a second stage, where the perturbations expand in spatial scale and increasingly come into geostrophic balance, and finally a third stage where the balanced perturbations grow on synoptic scales in the presence of a baroclinic instability. The growth rates of disturbance total energy (DTE) in the first and third stages, as well as the transition time between these phases were quantified by fitting a simple mathematical equation in (4) with three time scales. We found $e$-folding times for the initial growth on small spatial scales of $0.61 \mathrm{~h}$ and, after upscale propagation, of $12 \mathrm{~h}$ on large spatial scales. An exponential decay parameter was used to estimate a time for the saturation of the small-scale errors of about $11 \mathrm{~h}$.
Inspection of the error fields showed that initial growth was confined to regions where precipitation was occurring. Computing growth rates separately for precipitating and nonprecipitating regions confirmed that growth was much faster in the precipitating regions ( $e$-folding time: $0.34 \mathrm{~h}$ ). Using a threshold value of CAPE to further separate the precipitating regions into convective and stratiform areas showed that error growth was significantly faster for convective systems (Fig. 8). However, saturation occurred more quickly in convective regions and the final amplitude of the small-scale perturbations was found to be related to the precipitation rate, rather than the initial error growth rate or the conditional instability.

Since the precipitating regions where the errors initially grow occupy only a small fraction of the domain, the Fourier decomposition of the error in the first stage projects mainly onto the large scales. Consistently, growth rate and Rossby number are initially similar on all scales. However, this projection was not sufficiently large in amplitude to explain the subsequent growth of large-scale error at the observed exponential growth rate. Upscale transfer of energy is required. Plots and animations of the disturbance energy showed that the perturbations spread out from the convective regions at a speed consistent with that of a deep (troposphere filling) gravity wave. The time scale that would be required in order to spin up a geostrophically balanced response was estimated to equal the time required for such a gravity wave to cross the Rossby radius of deformation. We showed that the saturation time for the small-scale error growth was approximately equal to this time scale and, more importantly, we computed a Rossby number from the ratio of divergence and rotation, which showed that the large-scale perturbation also started to come into balance on this time scale.

The upscale transfer of energy was also reflected in a steepening of the disturbance kinetic energy spectrum in the mesoscale range $(200-1000 \mathrm{~km})$. However, this does not necessarily imply scale interactions in a cascade as in homogeneous turbulence theory. Perturbations propagating as waves through the nonprecipitating regions may have little interaction with the surrounding flow on the mesoscale and might only extract energy after reaching the synoptic scale where baroclinic instability can be excited. It is an important challenge to find a theoretical model of this process that takes into account the important phase information that is lost in a spectral energy density representation.

This study was based on a single period in the summer and the results are likely to vary significantly in different weather regimes. An indicator of this comes from comparison with the results of Zhang et al. (2007), 
where the amplitude of the perturbations after only $36 \mathrm{~h}$ is approximately twice that found in our case after $60 \mathrm{~h}$. This may be related to differences in the saturation of the small scales in domain-integrated diagnostics (cf. our Fig. 4 and their Fig. 6a) which takes much longer in our case and is not complete even $60 \mathrm{~h}$ after perturbation. There are two aspects of the meteorological environment that are likely to contribute to these differences. First, since error growth properties are very different in wet and dry areas their proportion in the domain may be important. The idealized baroclinic wave used in the study of Zhang et al. (2007) produced a relatively large-scale and steady precipitation field while in our real case the precipitation field is more intermittent [i.e., spottier, unsteady, and covering a much smaller area; cf. Zhang et al. (2007) their Fig. 4 and our Fig. 1]. Second, the idealized baroclinic wave was designed to represent a rapidly growing cyclone in its early stages, while in our case, despite the high convective activity, the synoptic-scale development is relatively weak.

The magnitude of the mean 500-hPa geopotential difference $60 \mathrm{~h}$ after perturbing was about half the spread (standard deviation) of the ECMWF 6-h ensemble forecast for this region and time. This suggests that the intrinsic predictability quantified in this study may play a significant, although perhaps not dominant, role in limiting the forecast skill. In general, the importance of upscale error growth in a numerical weather prediction system will depend on many practical details in addition to the intrinsic predictability. In a recent paper, Durran and Gingrich (2014) showed that for ensemble forecasts of two growing winter storms, the spread of the initial conditions from an ensemble Kalman filter have a substantial large-scale component that grows in the forecast ensemble without the need for significant upscale transfer of energy. These intense winter storms are of course very different from the summer case considered here, which has a relatively stationary synoptic pattern. In contrast, Rodwell et al. (2013) related an episode of bad weather forecasts and low predictability over Europe to strong convective activity over the eastern United States together with a trough over the Rocky Mountains. This study suggests that upscale error growth can indeed be of significant practical importance. However, it also indicates that it might well be highly regime dependent and nonlocal, meaning that at a given location it is not the in situ convection that is important in limiting predictability but the convection far upstream.

Some further information on the relative sensitivity of ensemble forecasts to large-scale versus small-scale uncertainty can be obtained from the study of Keil et al. (2014), who analyzed the skill of precipitation forecasts from the operational COSMO-DE-EPS high-resolution ensemble through the summer of 2009. In this ensemble, large-scale uncertainty is represented by using boundary conditions from four different global models, and small-scale uncertainty is included through five different configurations of the subgrid parameterization schemes, for a total of 20 ensemble members. In cases of synoptically forced convection, the large-scale boundary conditions dominate the ensemble spread, while for periods of weakly forced convection driven more by local instability, the contributions from both sources are approximately equal.

The experiments in this study extended only up to 60-h perturbation lead time because of computational costs that limited the domain size. At longer times the unperturbed lateral boundary conditions would increasingly suppress further error growth by imposing a maximum spatial scale and allowing perturbations to be advected out of the domain. However, the diagnostics and interpretation presented here suggest how the error growth would likely continue. Baroclinic instability would continue to increase the synoptic-scale error and thus further steepen the difference spectrum, but eventually saturate because of a complete displacement of high and low pressure systems and Rossby waves. The difference spectra will then probably equal 2 times the full state kinetic energy spectrum as expected for a complete phase decorrelation of the modes on all scales. The difference Rossby number will further decrease until it reaches the synoptic-scale value of about 0.1. Domain-integrated DTE on large scales will continue to grow exponentially for a time at the synoptic-scale growth rate (similar to $g$ ) and will also eventually saturate, probably on a time scale of a few weeks. To confirm this scenario would, however, require experiments in a global model.

Previous studies (Zhang et al. 2003; Tan et al. 2004) have shown that the initial error growth in model setups with parameterized convection is much slower than in high-resolution models. Since the resolution of global ensemble forecasting systems is still some distance from convection permitting this suggests an intrinsic overconfidence of such models with respect to upscale error growth from convective-scale uncertainty. This may be one of the reasons why global ensemble forecasts become underdispersive when only the initial state uncertainty is sampled. This shortcoming is currently addressed with more or less pragmatic approaches that introduce stochastic perturbations into the model [e.g., the stochastic perturbation of physical tendencies scheme (SPPT; Buizza et al. 1999) or the stochastic kinetic energy backscatter scheme (SKEB; Berner et al. 2009)]. These methods are tuned to make the ensemble system reliable, which requires the introduction of quite large spatial and temporal correlations in the perturbation structures. 
Upscale error growth processes as described in our study can be regarded as a potential justification for the need for stochastic perturbations on a broad range of space and time scales, rather than only at the smallest resolved scales.

In a subsequent study we will investigate if a stochastic convection scheme like Plant and Craig (2008), which uses a physically based distribution to randomly locate convective clouds, could be used to simulate the upscale error growth without having to resolve the convection. This, in turn, would enable the running of global ensemble forecasts to estimate the possible contribution of upscale error growth from convection to medium-range forecast uncertainty.

Acknowledgments. This project was supported by the German Research Foundation (DFG) as part of the research unit PANDOWAE (FOR896). Acknowledgement is made for the use of ECMWF's computing and archive facilities. We are also grateful to DWD for providing the COSMO model and especially to Ulrich Blahak and Ulrich Schättler for their help and support with it.

\section{REFERENCES}

Baldauf, M., A. Seifert, J. Förstner, D. Majewski, M. Raschendorfer, and T. Reinhardt, 2011: Operational convective-scale numerical weather prediction with the COSMO model: Description and sensitivities. Mon. Wea. Rev., 139, 3887-3905, doi:10.1175/ MWR-D-10-05013.1.

Berner, J., G. Shutts, M. Leutbecher, and T. Palmer, 2009: A spectral stochastic kinetic energy backscatter scheme and its impact on flow-dependent predictability in the ECMWF ensemble prediction system. J. Atmos. Sci., 66, 603-626, doi:10.1175/2008JAS2677.1.

Bierdel, L., P. Friederichs, and S. Bentzien, 2012: Spatial kinetic energy spectra in the convection-permitting limited-area NWP model COSMO-DE. Meteor. Z., 21, 245-258, doi:10.1127/ 0941-2948/2012/0319.

Bretherton, C. S., and P. K. Smolarkiewicz, 1989: Gravity waves, compensating subsidence and detrainment around cumulus clouds. J. Atmos. Sci., 46, 740-759, doi:10.1175/1520-0469(1989)046<0740: GWCSAD $>2.0 . \mathrm{CO} ; 2$.

Buizza, R., M. Milleer, and T. Palmer, 1999: Stochastic representation of model uncertainties in the ECMWF ensemble prediction system. Quart. J. Roy. Meteor. Soc., 125, 2887-2908, doi:10.1002/qj.49712556006.

Done, J. M., G. C. Craig, S. L. Gray, P. A. Clark, and M. E. B. Gray, 2006: Mesoscale simulations of organized convection: Importance of convective equilibrium. Quart. J. Roy. Meteor. Soc., 132, 737-756, doi:10.1256/qj.04.84.

Durran, D. R., and M. Gingrich, 2014: Atmospheric predictability: Why butterflies are not of practical importance. J. Atmos. Sci. 71, 2476-2488, doi:10.1175/JAS-D-14-0007.1.
_ P. A. Reinecke, and J. D. Doyle, 2013: Large-scale errors and mesoscale predictability in Pacific Northwest snowstorms. J. Atmos. Sci., 70, 1470-1487, doi:10.1175/JAS-D-12-0202.1.

Errico, R. M., 1985: Spectra computed from a limited area grid. Mon. Wea. Rev., 113, 1554-1562, doi:10.1175/1520-0493(1985)113<1554: SCFALA $>2.0 . \mathrm{CO} ; 2$.

Gill, A. E., 1982: Atmosphere-Ocean Dynamics. Academic Press, $261 \mathrm{pp}$.

Hohenegger, C., and C. Schär, 2007a: Atmospheric predictability at synoptic versus cloud-resolving scales. Bull. Amer. Meteor. Soc., 88, 1783-1793, doi:10.1175/BAMS-88-11-1783.

$\longrightarrow$, and — 2007b: Predictability and error growth dynamics in cloud-resolving models. J. Atmos. Sci., 64, 4467-4478, doi:10.1175/ 2007JAS2143.1.

Keil, C., F. Heinlein, and G. C. Craig, 2014: The convective adjustment time-scale as indicator of predictability of convective precipitation. Quart. J. Roy. Meteor. Soc., 140, 480-490, doi:10.1002/qj.2143.

Leoncini, G., R. S. Plant, S. L. Gray, and P. A. Clark, 2010: Perturbation growth at the convective scale for CSIP IOP18. Quart. J. Roy. Meteor. Soc., 136, 653-670, doi:10.1002/qj.587.

Leutbecher, M., and T. N. Palmer, 2008: Ensemble forecasting. J. Comput. Phys., 227, 3515-3539, doi:10.1016/j.jcp.2007.02.014.

Lorenz, E. N., 1969: The predictability of a flow which possesses many scales of motion. Tellus, 21, 289-307, doi:10.1111/ j.2153-3490.1969.tb00444.x.

, 1996: Predictability: A problem partly solved. Proc. Seminar on Predictability, Vol. 1, Reading, United Kingdom, ECMWF, $1-18$.

Melhauser, C., and F. Zhang, 2012: Practical and intrinsic predictability of severe and convective weather at the mesoscales. J. Atmos. Sci., 69, 3350-3371, doi:10.1175/JAS-D-11-0315.1.

Plant, R. S., and G. C. Craig, 2008: A stochastic parameterization for deep convection based on equilibrium statistics. J. Atmos. Sci., 65, 87-105, doi:10.1175/2007JAS2263.1.

Rodwell, M. J., and Coauthors, 2013: Characteristics of occasional poor medium-range weather forecasts for Europe. Bull. Amer. Meteor. Soc., 94, 1393-1405, doi:10.1175/BAMS-D-12-00099.1.

Tan, Z.-M., F. Zhang, R. Rotunno, and C. Snyder, 2004: Mesoscale predictability of moist baroclinic waves: Experiments with parameterized convection. J. Atmos. Sci., 61, 1794-1804, doi:10.1175/ 1520-0469(2004)061<1794:MPOMBW > 2.0.CO;2.

Wulfmeyer, V., and Coauthors, 2011: The Convective and Orographically-induced Precipitation Study (COPS): The scientific strategy, the field phase, and research highlights. Quart. J. Roy. Meteor. Soc., 137, 3-30, doi:10.1002/qj.752.

Zhang, F., C. Snyder, and R. Rotunno, 2003: Effects of moist convection on mesoscale predictability. J. Atmos. Sci., 60, 1173-1185, doi:10.1175/1520-0469(2003)060<1173:EOMCOM >2.0.CO;2.

— A. M. Odins, and J. W. Nielsen-Gammon, 2006: Mesoscale predictability of an extreme warm-season precipitation event. Wea. Forecasting, 21, 149-166, doi:10.1175/WAF909.1.

— , N. Bei, R. Rotunno, C. Snyder, and C. Epifanio, 2007: Mesoscale predictability of moist baroclinic waves: Convectionpermitting experiments and multistage error growth dynamics. J. Atmos. Sci., 64, 3579-3594, doi:10.1175/JAS4028.1.

Zhu, H., and A. Thorpe, 2006: Predictability of extratropical cyclones: The influence of initial condition and model uncertainties. J. Atmos. Sci., 63, 1483-1497, doi:10.1175/ JAS3688.1. 